\title{
ERRATUM
}

\section{Erratum to: Familial Cultural Values, Depressive Symptoms, School Belonging and Grades in Latino Adolescents: Does Gender Matter?}

\author{
Alexandra M. Cupito • Gabriela L. Stein • \\ Laura M. Gonzalez
}

Published online: 11 December 2014

(C) Springer Science+Business Media New York 2014

\section{Erratum to: J Child Fam Stud \\ DOI 10.1007/s10826-014-9967-7}

The original version of this article unfortunately contained a mistake. Table 4 was incorrect. The corrected Table 4 is given below. The authors apologize for this error and the inconvenience it has caused.

Table 4 Regression results of familial cultural values and gender predicting depressive symptoms, school belonging, and grades

\begin{tabular}{|c|c|c|c|}
\hline & $\begin{array}{l}\text { Familism } \\
\text { (A) } \\
\beta\end{array}$ & $\begin{array}{l}\text { Filial obligation } \\
\text { (A) } \\
\beta\end{array}$ & $\begin{array}{l}\text { Affiliative } \\
\text { obedience (A) } \\
\beta\end{array}$ \\
\hline \multicolumn{4}{|c|}{ Dependent variable: depressive symptoms } \\
\hline Cultural value (A) & $-.23 *$ & $-.48 * * *$ & $-.30 * *$ \\
\hline Age & .05 & .06 & .11 \\
\hline Gender (B) & $-.16^{*}$ & $-.17 *$ & -.13 \\
\hline $\mathrm{A} \times \mathrm{B}$ & .05 & $.30 * *$ & $.21 *$ \\
\hline Total $\mathrm{R}^{2}$ & .05 & $.06 *$ & $.04 *$ \\
\hline \multicolumn{4}{|c|}{ Dependent variable: school belonging } \\
\hline Cultural value (A) & $.27 *$ & $.40 * *$ & .16 \\
\hline Age & $-.14 *$ & -.14 & -.21 \\
\hline Gender (B) & -.06 & -.05 & -.10 \\
\hline $\mathrm{A} \times \mathrm{B}$ & .02 & -.06 & .02 \\
\hline Total $\mathrm{R}^{2}$ & .11 & .14 & .08 \\
\hline
\end{tabular}

The online version of the original article can be found under doi:10. 1007/s10826-014-9967-7.

A. M. Cupito $(\bowtie) \cdot$ G. L. Stein · L. M. Gonzalez

Department of Psychology, University of North Carolina

Greensboro, Greensboro, NC 27403, USA

e-mail: amcupito@uncg.edu

Table 4 continued

\begin{tabular}{|c|c|c|c|}
\hline & $\begin{array}{l}\text { Familism } \\
\text { (A) } \\
\beta\end{array}$ & $\begin{array}{l}\text { Filial obligation } \\
\text { (A) } \\
\beta\end{array}$ & $\begin{array}{l}\text { Affiliative } \\
\text { obedience (A) } \\
\beta\end{array}$ \\
\hline \multicolumn{4}{|c|}{ Dependent variable: grades } \\
\hline Cultural value $(\mathrm{A})$ & .05 & .13 & -.09 \\
\hline Age & .01 & .01 & .01 \\
\hline Gender (B) & $-.27 * * *$ & $-.32 * * *$ & $-.30 * * *$ \\
\hline $\mathrm{A} \times \mathrm{A}$ & - & -.21 & - \\
\hline $\mathrm{A} \times \mathrm{B}$ & .15 & .04 & .11 \\
\hline$A \times A \times B$ & - & .18 & - \\
\hline Total $\mathrm{R}^{2}$ & .10 & .13 & .10 \\
\hline
\end{tabular}

